\title{
ARTÉRIAS E SEGMENTOS ANATOMOCIRÚRGICOS DA GLÂNDULA ADRENAL EM FETOS DE BOVINOS
}

\author{
ARTERIES AND ANATOMICOSURGICAL SEGMENTS \\ OF THE ADRENAL GLANDS IN BOVINES FETUSES
}

Maria Angélica MIGLINO'; Liberato João Afonso DIDIO² ; João Batista da COSTAª Yolanda Galindo PACHECO

\begin{abstract}
RESUMO
As artérias e os segmentos anatomocirúrgicos das glândulas adrenais foram estudados em 30 fetos de bovinos, mediante o preenchimento do sistema arterial de cada animal com látex Neoprene 650 coradoe dissecação. A adrenal direita é vascularizada por 8 vasos diferentes, e à esquerda por 10 fontes diversas, com arranjos próprios em ambos os lados. $\mathrm{O}$ número de segmentos anatomocirúrgicos variou de $5 \mathrm{a} 1 \mathrm{l}$, sendo que à direita o número mais freqüente foi $3(60,0 \%)$ e à esquerda $3(33,3 \%)$ e $4(30,0 \%)$.
\end{abstract}

UNITERMOS: Artérias; Glândula adrenal; Bovinos

\section{INTRODUÇÃO}

Diante do nosso interesse em reconhecer a eventual existência de segmentos anatomocirúrgicos e diante da lacuna que se nota na literatura especializada sobre os vasos que nutrem a glândula adrenal, resolvemos estudar sua anatomia em bovinos.

Nossa intenção foi, pois, a de determinar a origem do suprimento sangüineo da glândula adrenal e a disposição das artérias no seu parênquima, com vistas a identificar uma segmentação vascular do órgão.

\section{MATERIAL E MÉTODO}

Foram estudados 30 fetos de bovinos Nelore, em diferentes fases da gestação, entre 4 e 9 meses.

Esses fetos ( 15 machos e 15 fêmeas) tiveram a aorta e seus ramos injetados com látex Neoprene corado e foram fixados em solução aquosa de formol a $10 \%$. Em seguida, as adrenais foram dissecadas e seus vasos, extremamente finos, foram observados com lupa.

Fizemos um esquema da disposição dos vasos de cada glândula, que nos serviu de base para a apresentação dos resultados e para sua ilustração.

A exposição dos resultados segue a descrição de GODINHO et al.' (1981), considerando assim para ambas as adrenais as seguintes regiões: caudal (contígua ao pólo cranial do rim), medial (vizinha à aorta), lateral (oposta à aorta) e cranial (oposta ao pólo renal).

1 - Professor Associado - Faculdade de Medicina Veterinária e Zootecnia da USP-SP

- Professor Visitante - Escola Paulista de Medicina - SP

- Professor Assistente - Faculdade de Zootecnia - Universidade Federal do Rio Grande do Norte-RN

4 - Professor Adjunto - Faculdade de Ciências da Saúde - Universidade de Brasilia - DF

\section{RESULTADOS}

\section{Artérias da glândula adrenal direita}

A origem e o número das artérias da glândula adrenal direita foram os seguintes:

1. da aorta (artéria adrenal): 18 casos/30, ou seja, $60,0 \% \pm 8,9$. Desses casos, correspondentes a 10 machos e 8 fêmeas, 2 (machos) apresentaram 2 artérias e os 16 restantes $(8$ machos e 8 fềmeas), 53.3\% $\pm 9,1$, apresentaram uma únıca artéria.

2. da artéria renal direita: 15 casos $/ 30$, ou seja, $50,0 \% \pm 9,1$. Desses casos, correspondentes a 8 machos e 7 fêmeas, I (macho) apresentou 2 artérias e os 14 restantes $(7$ machos e 7 fêmeas) apresentaram uma única artéria.

3. da artéria renal acessória: uma única artéria em 1 caso/30, ou seja, $3,33 \pm 3,3$.

4. do ramo frênico-torácico: uma única artéria em I caso/30, ou seja, $3,33 \pm 3,3$.

5. da $1^{\text {a }}$ lombar: 17 casos/30, ou seja, $56,6 \% \pm 9,1$. Desses casos, correspondentes a 9 fêmeas e 8 machos, 1 (macho) apresentou 3 artérias, 3 ( 2 fêmeas e 1 macho) apresentaram 2 artérias e os 13 restantes ( 7 fêmease 6 machos) apresentaram uma única artéria.

6. da $2^{\text {a }}$ lombar: 5 casos $/ 30$, ou seja, 16,6\% \pm 6,8. Desses casos, correspondentes a 3 fềmeas e 2 machos, 1 (fềmea) apresentou 2 artérias e os restantes apresentaram vaso único.

7. da artéria costo-abdominal dorsal: 17 casos/30, ou seja, $56,6 \%$ $\pm 9,1$. Desses casos, correspondentes a 11 machos e 6 fềmeas, 1 (macho) apresentou 2 artérias e os demais mostraram único vaso. 
MIGLINO. M.A.; DIDIO, L.J.A.; COSTA, J.B.; PACHECO, Y.G. Attérias e segmentos anatomocirúrgicos da glândula adrenal em fetos de bovinos. Braz. J. vet. Res. anim. Sci. São Paulo, v.32, n.2, p.75-82, 1995

8. da artéria frênico-abdominal caudal: 19 casos/30, ou seja, $63,3 \% \pm 8,8$. Em todas as oportunidades verificou-se a presença de um único vaso.

\section{Artérias da glândula adrenal esquerda}

A origem e o número das artérias da glândula adrenal esquerda foram os seguintes:

1. da aorta (artéria adrenal): 20 casos $/ 30$, ou seja, $66,6 \% \pm 8,6$. Desses casos, correspondentes a 11 fêmeas e 9 machos, 1 (macho) apresentou 3 vasos, 3 (machos) apresentaram 2 vasos e o restante (11 fềmeas e 5 machos) apresentou 1 único vaso.

2. da artéria renal esquerda: 13 casos/30, ou seja, $43,3 \% \pm$ 9.1. Desses casos, correspondentes a 8 machos e 5 fềmeas, todos apresentaram 1 único vaso.

3. da artéria celíaca: 11 casos 30 , ou seja, $36,6 \% \pm 8,8$. Desses casos, correspondentes a 7 fêmeas e 4 machos, 3 (2 fêmeas e 1 macho) apresentaram 2 artérias e o restante (5 fêmeas e 3 machos), 1 único vaso.

4. do tronco celíaco-mesentérico: 1 caso/30, ou seja, 3,3\% 3.3. Neste caso, correspondente a 1 macho, verificamos 2 vasos dirigidos à glândula.

5. da 1 lombar: 25 casos $/ 30$, ou seja, $83,3 \% \pm 6,8$. Desses casos, correspondentes a 13 fêmeas e 12 machos, 1 (macho) apresentou 3 vasos, 2 (fêmeas) apresentaram 2 vasos e os demais (12 machos e 10 fêmeas) mostraram 1 único vaso.

6. da $2^{\text {a }}$ lombar: 8 casos $/ 30$, ou seja, $26,6 \% \pm 8,1$. Desses casos, correspondentes a 6 fêmeas e 2 machos, 1 (macho) apresentou 4 vasos e os demais apresentaram 1 único vaso.

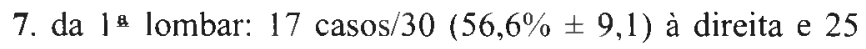
$\operatorname{casos} / 30(83,3 \% \pm 6,8)$ à esquerda. Dos casos da direita $(9$ fêmeas e 8 machos), 1 macho apresentou 3 artérias, 3 (2 fêmeas e 1 macho) apresentaram 2 artérias, e os 13 restantes ( 7 fềmeas e 6 machos) apresentaram 1 único vaso. À esquerda (13 machos e 12 fêmeas) 1 macho apresentou 3 artérias, 2 fềmeas apresentaram 2 vasos e os demais (12 machos e 10 fềmeas) mostraram 1 único vaso.

8 . da $2^{\mathrm{a}}$ lombar: 5 casos $(16,6 \% \pm 6,8)$ à direita e 8 casos $/ 30(26,6 \%$ $\pm 8,1$ ) à esquerda. À direita (3 fềmeas e 2 machos), 1 fềmea apresentou 2 artérias e os restantes ( 2 machos e 2 fềmeas) apresentaram 1 único vaso. À esquerda (6 fềmeas e 2 machos), 1 macho apresentou 4 vasos e os demais mostraram vaso único.

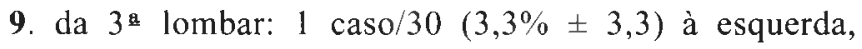
correspondente a 1 fêmea, na qual se verificou 1 único vaso.
10. da artéria costo-abdominal dorsal: 17 casos $/ 30(56,6 \% \pm$ $9,1)$ à direita e $2 \operatorname{casos} / 30(6,6 \% \pm 4,5)$ à esquerda. Dos 11 machos e 6 fêmeas à direita, 1 macho apresentou 2 artérias e os demais mostraram 1 único vaso. À esquerda em 2 machos, verificou-se a presença de 1 único vaso.

11. da artéria frênico-abdominal caudal: 19 casos $/ 30(63,3 \%$ $\pm 8,8)$ à direita e $1 \mathrm{caso} / 30(3,3 \% \pm 3,3)$ à esquerda, a qual (fêmea) apresentou 1 único vaso. À direita verificou-se também a presença de 1 único vaso nos 19 casos.

12. da artéria mesentérica cranial: 13 casos $/ 30(43,3 \% \pm 9,1)$, correspondentes a 8 machos e 5 fềmeas, somente à esquerda. Desses casos, 1 macho apresentou 3 vasos, 2 machos e 1 fêmea apresentaram 2 vasos e os demais ( 5 machos e 4 fêmeas) apresentaram 1 único vaso (Tab. 4).

\section{Segmentos anatomocirúrgicos da glândula}

Foram encontrados segmentos anatomocirúrgicos bilateralmente em todos os casos.

O número de segmentos foi igual bilateralmente em 7 casos de $30(23,3 \% \pm 7,7)$ e diferente em 23 casos $(76,7 \% \pm 7,7)$.

O número máximo de segmentos em cada caso (soma das glândulas adrenais direitas e esquerdas) foi de 11 (em 2 casos) e o mínimo foi de 5 ( 3 casos), de acordo com a Tab. 1.

\section{TABELA 1}

Número de segmentos arteriais anatomocirúrgicos da adrenal de bovinos e número de casos correspondentes. São Paulo, 1993.

\begin{tabular}{cc}
\hline $\mathrm{n}^{\mathbf{0}}$ de segmentos anatomocirúrgicos & $\mathrm{n}^{\mathrm{o}}$ de casos \\
\hline 05 & 03 \\
06 & 09 \\
07 & 10 \\
08 & 02 \\
09 & 03 \\
10 & 01 \\
11 & 02 \\
\hline
\end{tabular}

Nota-se que o ne total de segmentos mais freqüente é 7 ou 6 (bilateralmente), que ocorreram em $10(33,3 \% \pm 5,5)$ e 9 $(30,0 \% \pm 8,1)$ casos, respectivamente.

A igualdade e a desigualdade bilateral do número de segmentos foi notada em fêmeas e em machos (Quad. 1).

O número de segmentos foi maior à esquerda em 16 casos $(53,3 \%)$ e maior à direita em $7(47,7 \% \pm 7,7)$. 
O número de segmentos à direita variou de 2 (2 casos) a 6 ( 1 caso) e sua distribuição foi: (Tab. 2).

$\mathrm{O}$ número mais freqüente de segmentos da glândula adrenal

foi 3, que ocorreu em 18 casos $(60,0 \% \pm 8,9)$.

\section{QUADRO 1}

Segmentos anatomocirúrgicos da adrenal em bovinos. São Paulo, 1993.

Adrenal Esquerda

\begin{tabular}{|c|c|c|}
\hline № de Caso & No de Segmentos & Nome dos Segmentos \\
\hline 01 & 3 & 1 medial, I intermédio e 1 lateral \\
\hline 02 & 4 & 2 craniais, 1 caudomedial e 1 caudolateral \\
\hline 03 & 4 & 2 craniolaterais, 1 caudomedial e 1 ventral \\
\hline 04 & 6 & 2 craniais, I craniomedial, 1 craniolateral, 1 caudomedial e 1 caudolateral \\
\hline 05 & 4 & I cranial, 1 medial, 1 caudomedial e 1 caudolateral \\
\hline 06 & 5 & 2 craniais, 1 craniomedial, 1 caudomedial e 1 caudolateral \\
\hline 07 & 5 & l cranial, 1 craniomedial, 1 craniolateral, 1 medial e 1 caudolateral \\
\hline 08 & 6 & 2 craniais, 1 craniomedial, 1 craniointermédio, 1 craniolateral, 1 caudomedial e 1 caudolateral \\
\hline 09 & 4 & I cranial, 1 craniolateral, 1 caudomedial e 1 lateral \\
\hline 10 & 3 & 1 craniolateral, 1 craniointermédio e 1 craniolateral \\
\hline 11 & 2 & 1 cranial e 1 caudal \\
\hline 12 & 4 & 1 craniomedial, 1 craniointermédio, 1 craniolateral e 1 caudal \\
\hline 13 & 3 & 1 craniomedial, 1 craniointermédio e 1 craniolateral \\
\hline 14 & 4 & 1 cranial, 1 medial, 1 lateral e 1 caudal \\
\hline 15 & 4 & 1 cranial, 1 craniomedial, 1 craniolateral e 1 caudal \\
\hline 16 & 3 & 1 craniomedial, 1 craniolateral e 1 caudolateral \\
\hline 17 & 5 & 2 craniais, I craniomedial, 1 craniolateral e 1 caudal \\
\hline 18 & 4 & 1 craniomedial, 1 intermédio, 1 craniolateral e 1 caudal \\
\hline 19 & 2 & 1 cranial e 1 caudal \\
\hline 20 & 5 & 2 craniais, 1 craniolateral, 1 caudomedial e 1 caudal \\
\hline 21 & 8 & 3 craniais, 1 craniolateral, 1 craniointermédio, 2 mediais e 1 caudointermédio \\
\hline 22 & 2 & 1 craniolateral e 1 caudomedial \\
\hline 23 & 3 & 1 craniolateral, 1 caudomedial e 1 caudointermédio \\
\hline 24 & 3 & l craniomedial, 1 craniolateral e 1 caudolateral \\
\hline 25 & 3 & 1 craniomedial, 1 craniolateral e 1 caudal \\
\hline 26 & 3 & 1 craniolateral, 1 caudomedial e 1 caudolateral \\
\hline 27 & 2 & 1 cranial e 1 caudal \\
\hline 28 & 3 & I craniomedial, 1 craniolateral e 1 caudolateral \\
\hline 29 & 4 & 1 cranial, 1 craniomedial, 1 craniolateral e 1 caudomedial \\
\hline 30 & 3 & 1 craniomedial, 1 craniolateral e 1 caudomedial \\
\hline
\end{tabular}

TABELA 2

Número de segmentos arteriais anatomocirúrgicos da adrenal em bovinos e número de casos correspondentes. São Paulo, 1993.

\begin{tabular}{cc}
\hline $\mathrm{n}^{\circ}$ de segmentos anatomocirúrgicos & $\mathrm{n}^{\underline{0}}$ de casos \\
\hline 02 & 02 \\
03 & 18 \\
04 & 09 \\
06 & 01 \\
\hline
\end{tabular}

O número de segmentos à esquerda variou de 2 (4 casos) a 8 ( 1 caso) e sua distribuição foi: (Tab. 3). 
O número mais freqüente de segmentos da glândula adrenal esquerda foi 3 ou 4 , que ocorreram em $10(33,3 \%)$ e $9(30,0 \%)$ casos, respectivamente.

A distribuição dos casos pelo número de segmentos em cada lado é a seguinte: (Tab. 4)

\section{TABELA 4}

Número de segmentos arteriais anatomocirúrgicos em bovinos. São Paulo, 1993.

\begin{tabular}{cc}
\hline segmentos anatomocirúrgicos & $n^{\circ}$ de casos \\
\hline $2 D-3 E$ & 1 \\
$2 D-4 E$ & 1 \\
$3 D-2 E$ & 2 \\
$3 D-3 E$ & 6 \\
$3 D-4 E$ & 7 \\
$3 D-5 E$ & 1 \\
$3 D-6 E$ & 1 \\
$3 D-8 E$ & 1 \\
$4 D-2 E$ & 2 \\
$4 D-3 E$ & 3 \\
$4 D-4 E$ & 1 \\
$4 D-5 E$ & 2 \\
$4 D-6 E$ & 1 \\
$6 D-5 E$ & 1 \\
\hline
\end{tabular}

A disposição mais freqüente é a de 3 segmentos à direita e 4 à esquerda ( 7 casos ou $23,3 \% \pm 7,7)$ e a de 3 segmentos em cada lado (6 casos ou 20,0\% $\pm 7,3$ ) (Quad. 2).

\section{COMENTÁRIOS}

As artérias da adrenal de ruminantes foram estudadas por vários autores, tais como: HARRISON; McDONALD ${ }^{2}$ (1966); RUSSO; PELAGALLI ${ }^{3}$ (1972); GODINHO et al. ${ }^{1}$ (1981); TEWARI; PRAKASH ${ }^{4}$ (1988). Entretanto, os dois primeiros trabalhos foram dedicados aos pequenos ruminantes (ovinos $\mathrm{e}$ caprinos), razão pela qual comentaremos nossos resultados somente em função dos resultados encontrados por GODINHO et al. $^{1}$ (1981), e por TEWARI; PRAKASH ${ }^{4}$ (1988), que pesquisaram este assunto nos bovinos e bubalinos, respectivamente.

DeacordocomGODINHOetal. '( 1981),asglândulassupra-renais sãoirrigadas nos bovinos porramos das artérias frênicascaudais, celiaca e renais, sem entretanto especificar as percentagens da participaçãodestes vasosà direita eà esquerda. Apesarde termos encontradooitofontes diferentes deorigemdas artérias dirigidasà adrenal direitaedezàesquerda, aparticipaçãodasartérias frênicas caudaiserenais foiconstantenasnossasobservaçõestantoà direita $(63,3 \%$ e $50,0 \%$ respectivamente $)$, quanto à esquerda $(3,3 \% \mathrm{e}$ $43,3 \%$,respectivamente)
Quanto à participação de ramos derivados da artéria celíaca, este arranjo foi somente por nós observado à esquerda( $36,6 \%)$

No capítulo de resultados da pesquisa efetuada em bubalinos por TEWARI; PRAKASH ${ }^{4}$ (1988), para nossa surpresa, os autores, apesar de terem dissecado dez bezerros, referemse somente à origem e ramificações das artérias celíaca e mesentérica cranial, afirmando que, relativamente ao suprimento sangüíneo para a adrenal, existe para esta espécie uma evidente falta de informação sobre o assunto.

O número de vasos dirigidos à glândula, a comparação entre a vascularização das adrenais direita e esquerda bem como a segmentação do órgão não foram contemplados nas pesquisas que incluímos em nossa literatura.

\section{CONCLUSÕES}

1. As artérias adrenais, colaterais da aorta, contribuem para a vascularização da adrenal à direita e à esquerda.

2. As artérias renais direita e esquerda irrigam a adrenal em peças à direita e à esquerda.

3. Uma artéria renal acessória e uma frênico-torácica emitem ramo para a adrenal.

4. Em 1/3 dos casos, ramo derivado da artéria celíaca dirigese à adrenal. Quando as artérias celíaca e mesentérica cranial tinham a mesma origem (tronco celíaco-mesentérico), 2 vasos derivados deste tronco irrigavam a adrenal esquerda.

5. As primeiras artérias lombares, direita e esquerda, contribuem com a vascularização das adrenais, tanto à direita quanto à esquerda.

6. As segundas artérias lombares, direita e esquerda, também emitem ramos destinados às adrenais, à direita e à esquerda.

7. A terceira artéria lombar participa somente à esquerda em uma única ocasião, emitindo um ramo arterial para a adrenal.

8. A artéria costo-abdominal dorsal vasculariza a adrenal à direita e à esquerda.

9. As artérias frênico-abdominais caudais emitem ramo para as adrenais direita com grande freqüência, sendo remissivas à esquerda.

10. A artéria mesentérica cranial vasculariza a glândula adrenal esquerda.

11. Foram encontrados segmentos anatomocirúrgicos 
bilateralmente em todos os casos. Este número foi diferente bilateralmente na maioria dos casos. O número máximo de segmentos (soma das adrenais direita) foi 11 e o mínimo 5 , sendo o mais frequiente 7 ou 6.(bilateralmente).

12. O número de segmentos foi maior à esquerda $(53,3 \%) \mathrm{e}$ maior à direita em $47,7 \%$ dos casos. $\grave{A}$ direita, este número variou de 2 a 6 , mais freqüentemente 3 , e à esquerda, de 2 a 8 , freqüentemente 3 ou 4 casos, respectivamente. A disposição mais freqüente é a de 3 segmentos à direita e 4 à esquerda e a de 3 segmentos de cada lado.

QUADRO 2

Número de segmentos arteriais das adrenais em bovinos da raça Nelore. São Paulo, 1993.

Adrenal Direita

\begin{tabular}{|c|c|c|}
\hline Ne de Caso & $\mathrm{N}^{\alpha}$ de Segmentos & Nome dos Segmentos \\
\hline 01 & 2 & I craniolateral e 1 caudomedial \\
\hline 02 & 3 & 1 cranial, 1 caudomedial e 1 caudolateral \\
\hline 03 & 3 & 1 cranial, 1 caudomedial e 1 caudolateral \\
\hline 04 & 3 & 1 craniolateral, 1 caudomedial e 1 caudolateral \\
\hline 05 & 3 & 1 cranial, 1 caudomedial e 1 caudolateral \\
\hline 06 & 4 & 1 craniomedial, 1 craniolateral, 1 caudomedial e 1 caudolateral \\
\hline 07 & 3 & 1 cranial, 1 caudomedial e 1 caudolateral \\
\hline 08 & 4 & 1 cranial, 1 caudolateral, 1 caudomedial e 1 caudointermédio \\
\hline 09 & 4 & 1 cranial, 1 medial, 1 caudomedial e 1 caudolateral \\
\hline 10 & 3 & 1 craniolateral, 1 caudomedial e I caudolateral \\
\hline 11 & 3 & 1 craniomedial, 1 craniolateral e 1 caudal \\
\hline 12 & 3 & 1 craniolateral, 1 caudomedial e 1 caudolateral \\
\hline 13 & 4 & 1 cranial, 1 craniomedial, 1 caudomedial e 1 caudolateral \\
\hline 14 & 2 & 1 craniolateral e 1 caudomedial \\
\hline 15 & 3 & 1 cranial, I caudomedial e 1 caudolateral \\
\hline 16 & 3 & 1 craniolateral, 1 intermédio e 1 caudomedial \\
\hline 17 & 6 & 3 craniais, 1 intermédio, 1 caudomedial e 1 caudolateral \\
\hline 18 & 3 & 1 craniointermédio, 1 caudomedial e I caudolateral \\
\hline 19 & 4 & 1 cranial, 1 intermédio, 1 caudomedial e 1 caudolateral \\
\hline 20 & 4 & 1 craniomedial, 1 craniolateral, 1 intermédio e 1 caudomedial \\
\hline 21 & 3 & I craniolateral, I caudomedial e I caudolateral \\
\hline 22 & 4 & 1 craniomedial, 1 craniolateral, 1 medial e 1 intermédio \\
\hline 23 & 3 & 1 craniomedial, 1 craniolateral, 1 ventral \\
\hline 24 & 3 & 1 craniomedial, 1 craniolateral e 1 ventrointermédio \\
\hline 25 & 4 & 2 craniais, 1 craniolateral e 1 caudomedial \\
\hline 26 & 3 & 1 cranial, 1 lateral e 1 caudomedial \\
\hline 27 & 3 & 1 cranial, 1 lateral e I caudomedial \\
\hline 28 & 3 & 1 cranial, 1 lateral e 1 caudomedial \\
\hline 29 & 3 & 1 craniomedial, I craniolateral e 1 caudomedial \\
\hline 30 & 4 & 1 cranial, 1 medial, 1 lateral e 1 caudomedial \\
\hline
\end{tabular}


MIGLINO, M.A.: DIDIO, L.J.A.; COSTA, J.B.; PACHECO, Y.G. Arterias e segmentos anatomocirúrgicos da glândula adrenal em fetos de bovinos. Braz. J. vet. Res. anim. Sci. São Paulo, v.32, n.2, p.75-82, 1995 .

TABELA 5

Origem dos vasos arteriais penetrantes nas adrenais de bovinos de raça Nelore. São Paulo, 1993.

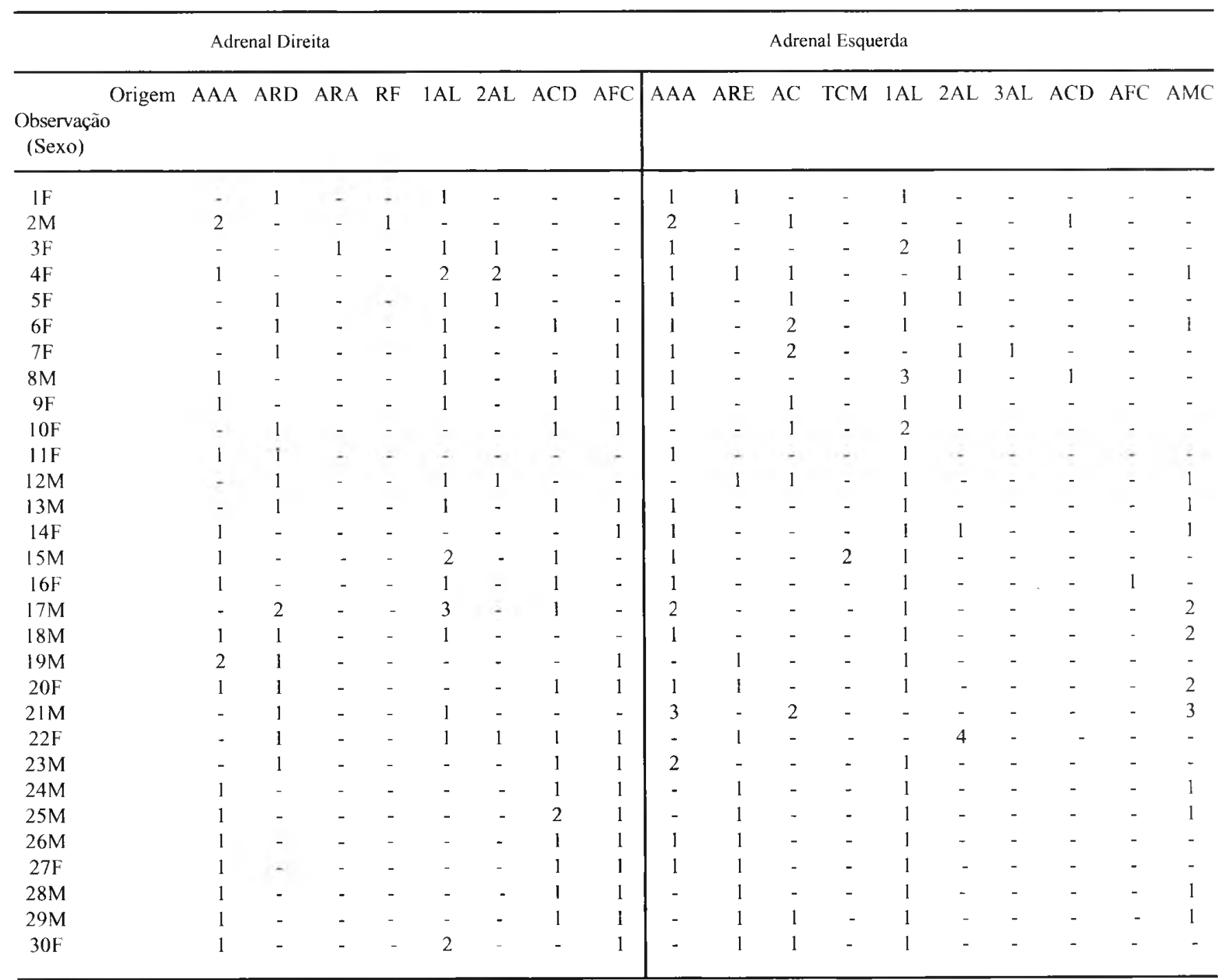

AAA - Aorta (Artéria Adrenal)

AC - Artéria Celíaca

ACD - Artéria Costo-Abdominal Dorsal

AFC - Artéria Frênico-Abdominal Caudal

AMC - Artéria Mesentérica Cranial

ARA - Artéria Renal Acessória

ARD - Artéria Renal Direita

ARE - Artéria Renal Esquerda

RF - Ramo Frênico-Torácico

TCM - Tronco Celíaco-Mesentérico

$1 \mathrm{AL}$ - $1 \stackrel{\text { B }}{\text { Artéria Lombar }}$

$2 \mathrm{AL}$ - 2 a Artéria Lombar

$3 \mathrm{AL}$ - $3^{\mathrm{a}}$ Artéria Lombar 


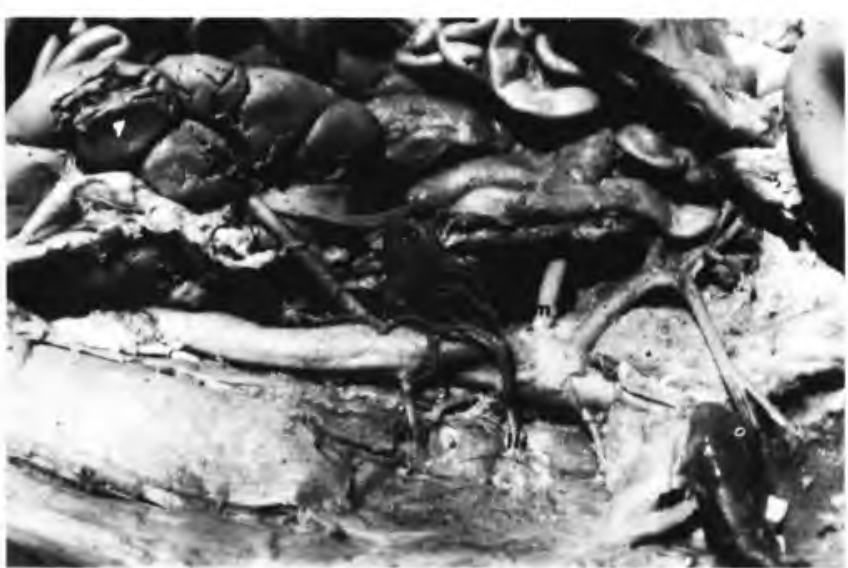

FIGURA 1

Fotografia de una peça dissecada correspondente a um feto de bovino da raça Nelore, onde se vê a adrenal direita (D) e a sua vascularização garantida por ramos derivados das artérias lombares (II e III), da artéria renal (r) e da artéria mesentérica cranial (m).

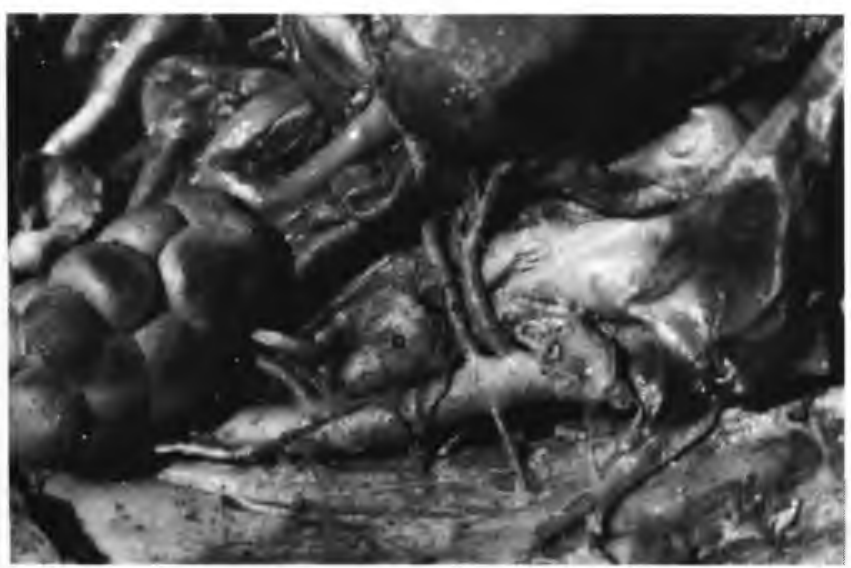

FIGURA 3

Fotografia da dissecação dos vasos endereçados à adrenal direita (D) de um feto de bovino da raça Nclore. Neste caso, a glândula recebe ramos arteriais oriundos das artérias celíaca (c), $2^{\underline{a}}$ lombar (II) e aorta (artéria adrenal) (a)

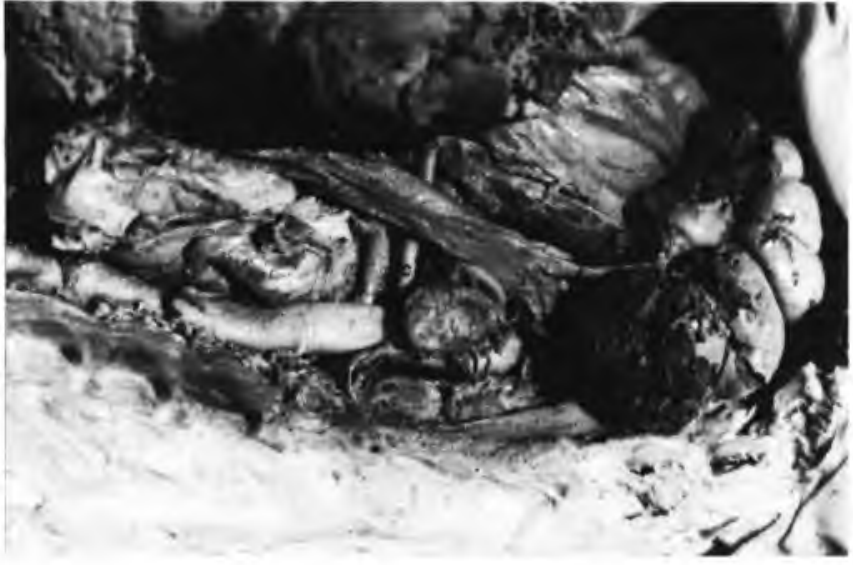

FIGURA 2

Fotografia de uma preparação correspondente a um feto de bovino da raça Nelore, onde se nota a adrenal recebendo vascularização das artérias lombares (I e II), da artéria celíaca (c) e da artéria frênicoabdominal caudal ( $f$ ).

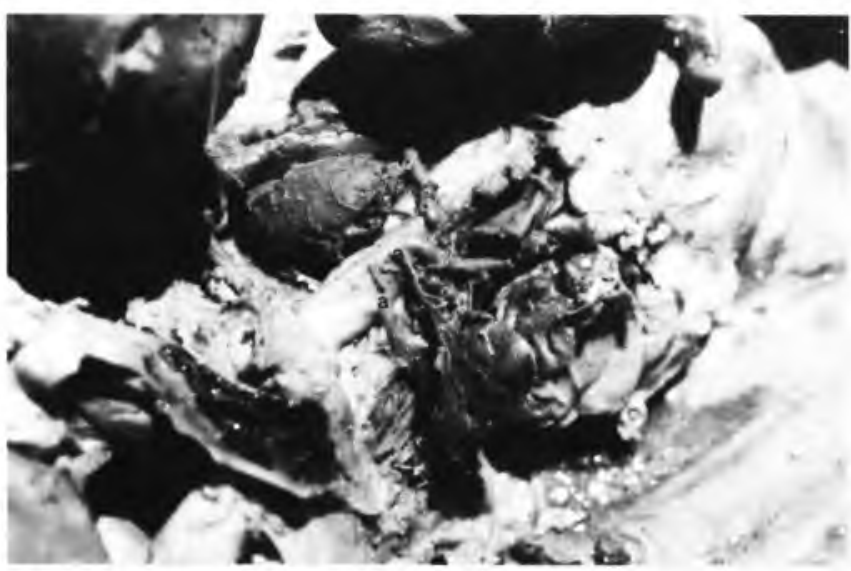

FIGURA 4

Fotografia de uma dissecação das artérias da adrenal esquerda onde a glândula recebe duas artérias adrenais (a), ramos colaterais da aorta.

\section{SUMMARY}

The arteries and the anatomicosurgical segments of the adrenal glands of 30 bovine fetuses were studied after arterial injection of Neoprene latex and dissection. The right adrenal gland may be supplied by 8 arteries and the left one by 10 , showing characteristic arrangements to each side. The number of anatomicosurgical segments varied from 5 to 11 . The most frequent number of segments in the right gland was $3(60 \%)$ and in the left one was $3(33.3 \%)$ or $4(30 \%)$.

UNITERMS: Arteries; Adrenal gland; Cattle 
MIGLINO. M.A.; DIDIO, L.J.A.; COSTA, J.B.; PACHECO, Y.G. Artérias e segmentos anatomocirúrgicos da glândula adrenal em fetos de bovinos. Braz. J. vet. Res. anim. Sci. São Paulo. v.32, n.2, p.75-82, 1995.

\section{REFERÊNCIAS BIBLIOGRÁFICAS}

1-GODINHO, H. P. ; CARDOSO, F. M. ; NASCIMENTO, J.F. Anatomia dos ruminantes domésticos. Belo Horizonte, Instituto de Ciências Biológicas da Universidade Federal de Minas Gerais, 1981.

2-HARRISON, F.A.; McDONALD, I.R. The arterial supply to the adrenal gland of the sheep. Journal of Anatomy, v. 100, n 91, p. 189-202, 1966.

3-RUSSO, E.; PELAGALLI, G.V. Macro-et-micro-circulation des glandes surrénales chez les petits ruminants. Recherches effectuées sur les espèces ovines et caprines. Acta anatomica, v. 82, p.179-97, 1972.
4-TEWARI, A.N.; PRAKASH, P. A note on the blood supply to the adrenal gland and spleen in the buffalo. Indian Veterinary Journal, v.65, p.270, 1988.

Recebido para publicação em 17/08/93 Aprovado para publicação em 12/09/94 